\title{
Study on SLT calibration method of 2-port waveguide DUT
}

\author{
Wenqiang Luo ${ }^{\mathrm{a}}$, Anyong $\mathrm{Hu}$, Kai Liu and Xi Chen \\ School of Electronics and Information Engineering, Beihang University, Beijing, 100083, China
}

\begin{abstract}
SSLT (Short-offset-Short-Load-Through) is a classical calibration method of VNA (Vector Network Analyser) which needs multiple assembly of the standard parts and exists the impact of the phase in the millimetre waveband. Aimed that the S11 of the two-port waveguide DUT is less than $-10 \mathrm{~dB}$, a simple SLT (Short-Load-Through) calibration method is presented in this paper according to the simplified error model. This calibration method only needs to carry on the calibration of short circuit plate and the matching load in the one-port of the VNA, and the through in the dual-port. The calibration process and the correction algorithm is simple. Finally, it is found that the amplitude relative error of the SLT calibration method is less than $2 \%$ and the phase calibration error is less than $2.5^{\circ}$ through the statistics of several DUTs.
\end{abstract}

Keywords: 2-port waveguide DUT; SSLT calibration; VNA.

\section{Introduction}

VNA (Vector Network Analyser) is one of the important measuring instruments of the DUT (Device Under Test) in the microwave or millimetre waveband. The calibration and correction of the error model in the VNA is the key to accurately measure the performance of the DUT [1]. For the 2-port waveguide DUT, it needs two or more fixtures to connect the waveguide port of the DUT and the coaxial port of the VNA. The S-parameters of those fixtures are consequently introduced into the measurements results as errors. So it's necessary to remove the effect of the test fixtures by the error calibration and correction of the VNA.

SOLT (Short-Open-Load-Through) calibration, a traditional VNA calibration method of the coaxial device, complete the four S-parameters calibration of the 2-port DUT by using the four standards (short-circuit, open-circuit, matching load and through). For the DUT of waveguide port, the calibration method and standards are related to the characteristics of the waveguide. The open circuit of the waveguide is equivalent to a poorly matching load, so it can't be replaced by the open waveguide. According to the theory of the microwave transmission line, short circuit plate with $\lambda / 4$ offset is equivalent to the open circuit. So short circuit plate with offset replace the waveguide standard of the open one [2]. Therefore, we call this calibration method SSLT (Short Offset Short Load Through) calibration instead of SOLT calibration.

SSLT calibration exists the problem of multiple assembly standard, that it needs three standards of the signal port and through calibration of the two-port. Furthermore, the offset range of short circuit plate becomes larger in the millimetre wave band. Additionally, the calibration algorithm is complex for testing [3-5]. Aimed that the S11 of the two-port waveguide DUT is less than -10dB, a simple SLT

\footnotetext{
a Corresponding author : luo_wq92@126.com
} 
(Short-Load-Through) calibration method is presented in this paper. This calibration method only needs to carry on the short circuit plate and the matching load calibration in the one-port of the VNA, and through calibration in the dual-port. Moreover, there is no impact on the actual phase with offset range.

This paper is organized as follows. Section II gives a comprehensive processing of the proposed SLT calibration. In section III, experimental results that verify the usefulness and accuracy of the proposed method are presented. Finally, Section IV gives general conclusions concerning the SLT calibration technique.

\section{SLT calibration}

12-error model is one of 2-port VNA calibration model that is extensively used in VNA calibration [6-8]. In this paper, 12-error model is simplified to 6-error model combined with the characteristics of 2port waveguide DUT. Error calibration and modified formula, namely the relationship between real value of the DUT and the measured value of the standard, is gotten based on the relationship of the error term in the signal flow graph.

\subsection{Error model}

12-error model is one of the most widely used error models in the three-channel two-port vector network analyser, which includes forward model and backward model, as shown in figure 1 . The system error includes the direction error $E_{D}$, the reverse tracking error $E_{R}$, the equivalent source mismatch error $E_{S}$, the difference error $E_{X}$, the forward tracking error $E_{T}$ and the load mismatch error $\mathrm{E}_{\mathrm{L}}$.

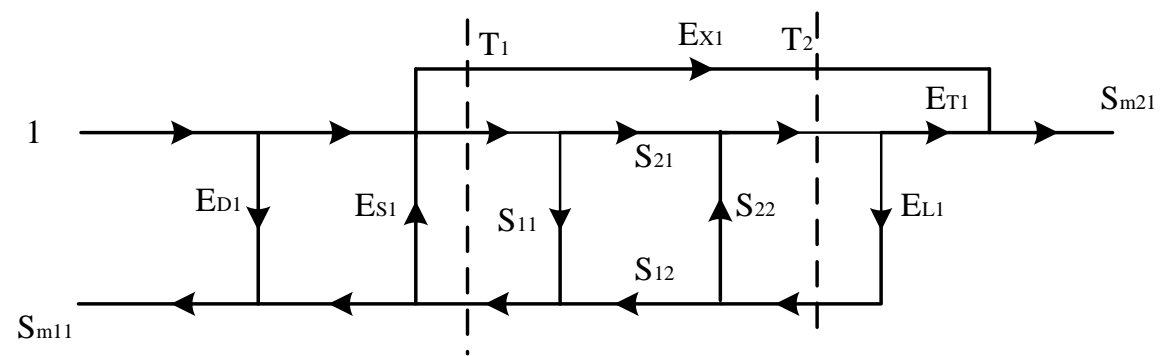

(a) Forward model

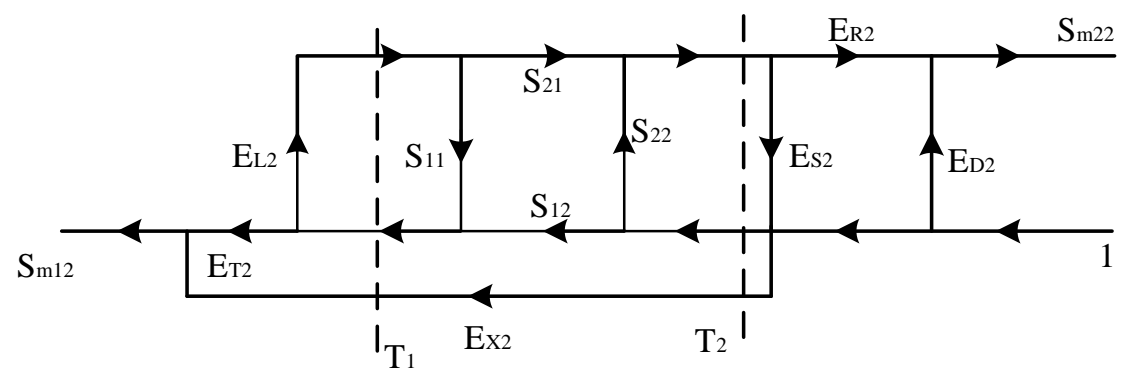

(b) Backward model

Figure 1. Schematic diagram of 12 error models

The calibration and correction is studied by taking the $S_{11}$ and $S_{21}$ as an example, and the calibration of $S_{22}$ and $S_{12}$ is similar to that of $S_{11}$ and $S_{21}$. Difference error $\left(E_{X}\right)$ is the leakage error of the source and receiver in the VNA. It is generally less than $-40 \mathrm{~dB}$ because of excellent hardware performance of commercial VNA. So the difference error $\left(E_{X}\right)$ can be ignored in the calibration process. Source and load mismatch error $\left(E_{S} / E_{L}\right)$ is cause by the multiple reflections of the incomplete matching in the port of incentive port or the non-incentive port of the DUT. In this test, the DUT has a 
high matching degree with the tested fixture, and the S11 of the DUT is less than -10dB [9-13]. So in this case, the source and load mismatch error $\left(E_{S} / E_{L}\right)$ can be ignored in the calibration process. Simplified 6-error model is gotten, as shown in figure2.

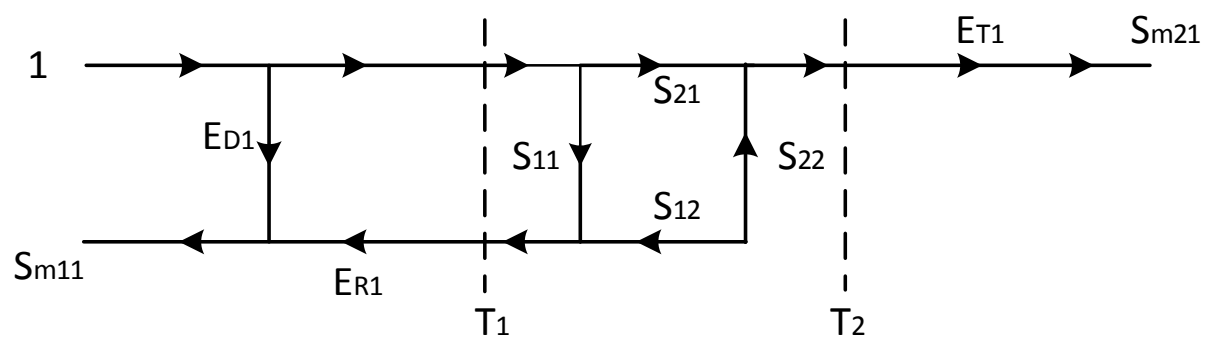

Figure 2. Schematic diagram of simplified 6-error model

\subsection{Error calibration}

SLT calibration includes the SL calibration of one-port (short circuit and matching load) and through calibration [14-16]. Specific calibration is as follows:

\subsubsection{SL calibration of one-port}

SL calibration is mainly calibrating the $S_{11}$ of the DUT. When the one-port of the VNA connects with the short circuit chip or the matching load, the error model can be simplified to a one-port error model, as shown in Figure3.

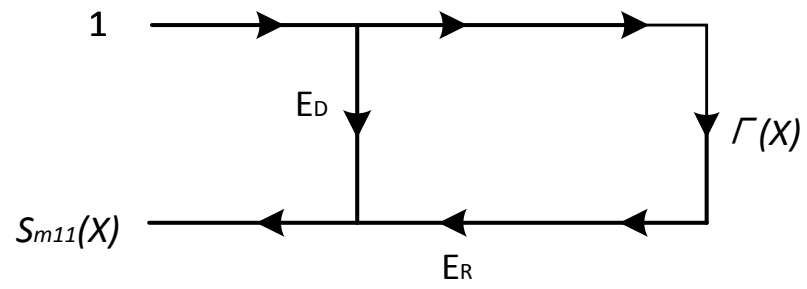

Figure 3. One-port error model

The expression of the relationship between the reflection coefficient and the corresponding measured value of the standard according to the signal flow graph:

$$
S_{m 11}(\mathrm{X})=E_{D}+E_{R} \cdot \Gamma(\mathrm{X})
$$

where $\Gamma(X)$ is the reflection coefficient of the standard $\mathrm{X}$, and $\mathrm{S}$ says the short circuit, and $\mathrm{L}$ says the matching load, and $\mathrm{S}_{\mathrm{m} 11}(\mathrm{X})$ is the corresponding measured values of the standard.

The following expression can be gotten when the short circuit and matching load is respectively connected to the one-port of VNA:

$$
\left\{\begin{array}{l}
S_{m 11}(L)=E_{D} \\
S_{m 11}(S)=E_{D}+E_{R}
\end{array}\right.
$$

The value of error term $E_{D}$ and $E_{R}$ of one-port can be obtained by equation group (2) as follows:

$$
\left\{\begin{array}{l}
E_{D}=S_{m 11}(L) \\
E_{R}=S_{m 11}(S)-S_{m 11}(L)
\end{array}\right.
$$




\subsubsection{Through calibration}

Through calibration is calibrating the $S_{21}$ of the DUT. In the through calibration, it supposes that $S_{11}$ and $S_{22}$ of the through connection equal to 0 , and $S_{12}$ and $S_{21}$ amount to 1 . The through calibration error model is shown as figure 4 .

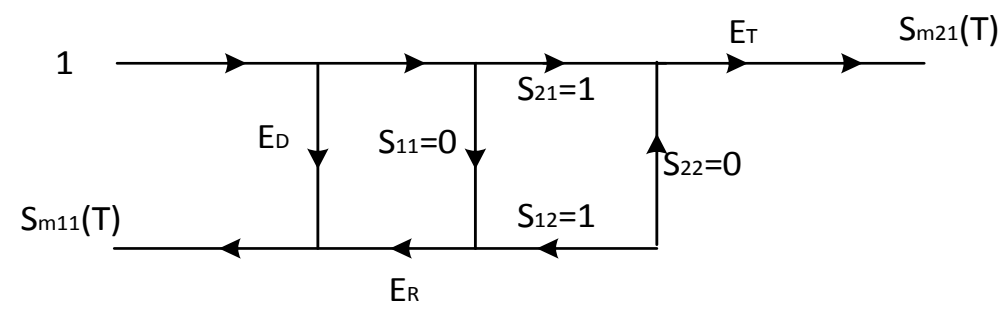

Figure4. Through calibration error model

The expression of the relationship between the error term and the corresponding measured value of the through according to the signal flow graph can be expressed as:

$$
S_{m 21}(\mathrm{~T})=E_{T}
$$

where $S_{m 21}(T)$ is the measured value of through calibration. The relationship between error terms and the standards can be obtained from that way. Meanwhile, the calibration of the 6-error model is completed.

\subsection{Error correction}

On the basis of error calibration, the influence of error term of VNA on the DUT is eliminated by error correction while the 2-port DUT is tested. Because the $S_{11}$ and $S_{21}$ of the 2-port waveguide DUT is tested, the error correction is only considered of the incentive of VNA Port1.

The following can be obtained from the forward model while the port1 is excited:

$$
\left[\begin{array}{c}
S_{m 11} \\
a_{1}
\end{array}\right]=\left[\begin{array}{cc}
E_{D 1} & E_{R 1} \\
1 & 0
\end{array}\right]\left[\begin{array}{l}
1 \\
b_{1}
\end{array}\right]
$$

So the incident wave $a_{1}$ and the reflected wave $b_{1}$ of port 1 can be expressed as:

$$
\left\{\begin{array}{l}
a_{1}=1 \\
b_{1}=\frac{S_{m 11}-E_{D 1}}{E_{R 1}}
\end{array}\right.
$$

Similarly, the incident wave $\mathrm{a}_{2}$ of port1 can be expressed as:

$$
a_{2}=\frac{S_{m 21}}{E_{T 1}}
$$

According to the definition of the S parameter and the expression (6) and (7), the corrected $S_{11}$ and $S_{21}$ can be obtained. Correction formulation can be gotten as follow while expression of error terms that are gotten from the error calibration are taken into the $S_{11}$ and $S_{21}$. 


$$
\left\{\begin{array}{l}
S_{11}=\frac{S_{m 11}-S_{m 11}(\mathrm{~L})}{S_{m 11}(\mathrm{~S})-S_{m 11}(\mathrm{~L})} \\
S_{21}=\frac{S_{m 21}}{S_{m 21}(\mathrm{~T})}
\end{array}\right.
$$

Among them, $\mathrm{S}_{\mathrm{m} 11}(\mathrm{~S})$ and $\mathrm{S}_{\mathrm{m} 11}(\mathrm{~L})$ are the measured data of $\mathrm{S} 11$ about the short circuit and matching load, and $S_{m 21}(T)$ is the measured data of $S_{21}$ about the through calibration, and $S_{m 11}$ and $S_{\mathrm{m} 21}$ is the tested data of $S_{11}$ and $S_{21}$ about the DUT, and the $S_{11}$ and $S_{21}$ is corrected data.

\section{Measurement and result}

The LNA (Low Noise Amplifier) is selected as the DUT in this paper, which of $S_{11}$ is less than -10dB. The comparison between the SLT calibration and SSLT calibration about the amplitude of $\mathrm{S}_{11}$ and amplitude and phase of $S_{21}$ is done following.

\subsection{Measurement of $S_{11}$}

SLT calibration is presented according that the source and load mismatch error $\left(E_{S} / E_{L}\right)$ are ignored, while it is based that the $S_{11}$ of the DUT is less than $-10 \mathrm{~dB}$. So the DUT we select is the LNA with $S_{11}$ less than $-10 \mathrm{~dB}$.

The $S_{11}$ testing result of SLT calibration and SSLT calibration is shown in figure5. It can be seen from the figure that the $S_{11}$ of the two calibrations are basically consistent.

Meanwhile, the average value of $S_{11}$ in $2 G$ bandwidth with 11 DUTs and the relative error of the two calibrations are counted, and the results are shown in Figure6. As can be seen from the figure, when the $S_{11}$ of the DUT is more than $-10 \mathrm{~dB}$, the error of two calibrations is relatively large, and while the $S_{11}$ of DUT is less than $-10 \mathrm{~dB}$, the relative error between two calibration is less than $2 \%$.

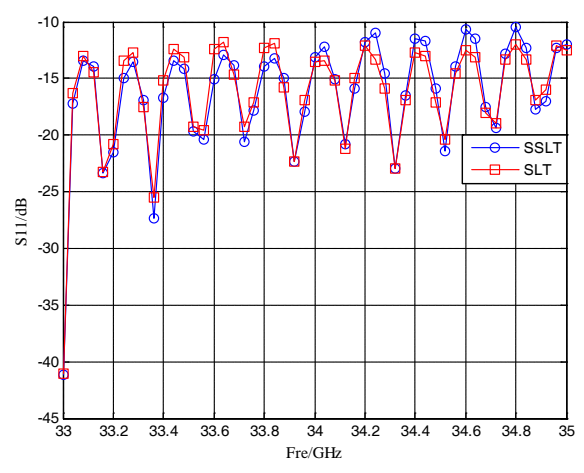

Figure5. Comparison of test results of SSLT and SLT calibration methods for $S_{11}$

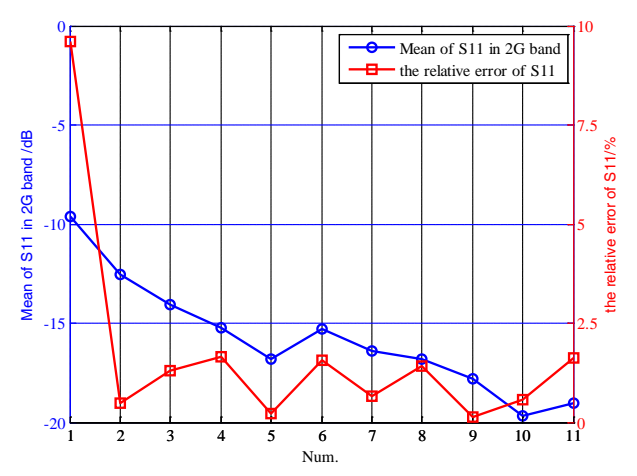

Figure6. Mean of $\mathrm{S}_{11}$ in $2 \mathrm{G}$ band and relative error statistics of two calibration methods

\subsection{Measurement of $S_{21}$}

The $\mathrm{S}_{21}$ testing result of SLT calibration and SSLT calibration is shown in figure7, where the graph (a) is the S21 amplitude result, and the graph of (b) is the S21 phase result. It can be seen from the figure that the S21 of the two calibrations are basically consistent.

Meanwhile, the average value of $S_{21}$ in 2G bandwidth of 11 DUTs and the relative error of the two calibrations are counted, and the results are shown in Figure8. As can be seen from the figure8(a), the relative error of the average value of $S_{21}$ in $2 G$ bandwidth between two calibrations is less than $2 \%$. At the same time, the relative error of the S21 phase of the two calibrations is less than $2.5^{\circ}$. 


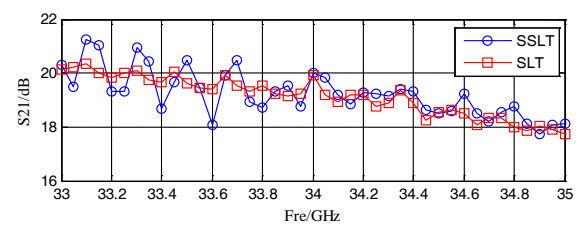

(a) Amplitude

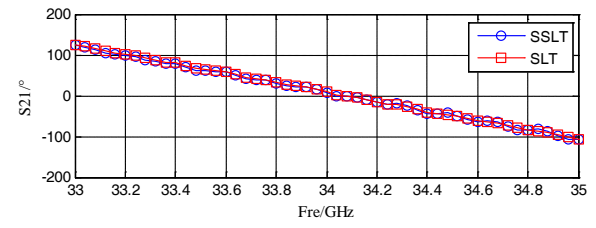

(b) Phase

Figure7. Comparison of test results of SOLT and SLT calibration methods for $\mathrm{S}_{21}$

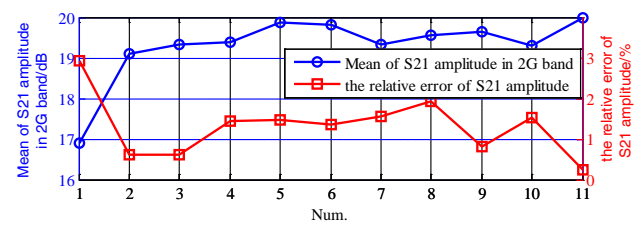

(a) Amplitude

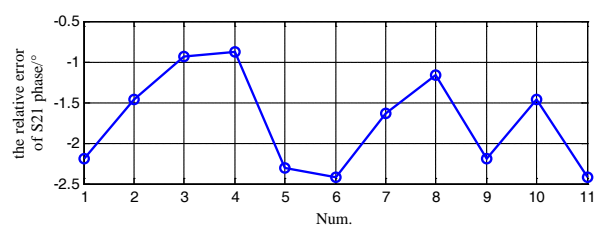

(b) Phase

Figure8. Mean of $\mathrm{S}_{21}$ in $2 \mathrm{G}$ band and relative error statistics of two calibration method

\section{Conclusion}

The SLT calibration technique, which uses short circuit, matching load and through as calibration elements, has been introduced and its usefulness and precision has been experimentally demonstrated. This calibration method do not need multiple assembly standard. The calibration and the correction algorithm is simple. Through the measurement, it can be seen that the relative error of the magnitude of $S_{11}$ is below $2 \%$, and the relative error of the magnitude of $S_{21}$ is less than $2 \%$, the phase error is less than $2.5^{\circ}$. It meet the accuracy requirements of the test, and verify the correctness and reliability of the SLT calibration. This calibration can be extended to the field of automated testing for quickly testing.

\section{References}

1. Horibe Masahiro, Kishikawa Ryoko. Measurement uncertainty in waveguide VNA calibrated by offset short calibration with oversized waveguide aperture at sub-millimetre wave frequency. Microwave Measurement Conference (ARFTG), 2014 84th ARFTG, page(s): 1-4

2. Zhao X, Study on Reflection Coefficient Measurement in Quasi-optical Millimeter-wave System Front-end, BUAA, (2011)

3. M. Horibe, et. al. Complete characterization of rectangular waveguide measurement standards for vector network analyzer in the range of millimeter and sub-millimeter wave frequencies. 76th ARFTG Conf. Digest, pp. 1-15, Dec. (2010).

4. Li W, Wang Z G, Li Z Q. Comparison of calibration methods of SOLT and TRL in S parameter measurement and calibration[C]. Proceedings of the Fifth China Test Conference.5.111-115, (2008)

5. A.A.Savin. A novel factor verification technique for one-port vector network analyzer. 43rd European Microwave Conference, Oct. (2013).

6. Li E, Xiang Z J, Guo G F, Zhang Q X, Research on TRL calibration technique of ridge waveguide, Journal of instrument and meter, 09:1041-1043, (2006).

7. Zhao W, Research on Calibration Technology of multi port vector network analyzer[D], NUAA, (2011).

8. Yuan C H. Calibration and correction of multi port vector network analyzer[D], NUAA, (2012)

9. Qin H B, Research on Calibration Technology of vector network analyzer, Xidian University, (2014) 
10. Quan L Y, Research on calibration method of vector network analyzer, Electronic quality, 2010,03:61-63.

11. Liu H, Calibration method of vector network analyzer, Electronic quality, 2011,07:71-72.

12. Ye R F, Xu J, SOLT calibration method and its application in radio frequency measurement, Electronic quality, 2006,01: 179-182.

13. Yuan C H, Zhao W, Zhao Y J, Liu B, Qiang L, A SOLT calibration method based on 10 error models of T matrix [J], Computer and digital engineering, 2011,11:10-12.

14. J. Stenarson. Residual error models for the SOLT and SOLR VNA calibration algorithms. in Submitted to 69th ARFTG Conference, (Honolulu Hawaii), 2007.

15. Agilent, Applying error correction to network analyzer measurement, Tech. Rep. AN 1287-3, 2002.

16. Stenarson, J. Eio, C. Yhland, K. A calibration procedure for electronic calibration units, Microwave Measurement Conference (ARFTG), 2014 84th ARFTG, On page(s): 1- 6 